Editorial

\title{
Editorial for the special section on Software Product Line Engineering: Selected papers from Software Product Line conference in 2012
}

"In theory, there is no difference between theory and practice. But, in practice, there is." (anonymous) is a famous saying that subsumes the difficulties in transferring research results to practice. Software engineering is one of the fields that close the gap more easily. This is definitely true for the publications in this special edition. Optimizing the economic benefit of reuse for a family of products is the core motivation for Software Product Line Engineering (SPLE). This consequently leads to a set of problems that involve several or all of the following: big systems and/or portfolios, considerable complexity due to variability, many involved stakeholders and long-lived legacy. Strategic reuse conferences have strong industry participation because SPLE research has to deal with complex, "real world" challenges.

The three articles presented here differ in the concrete challenges they solve, but are similar in their aim to generate economic benefit in a complex setting by increasing efficiency. The first article describes a framework for efficient management of product lines in a very specific horizontal domain, the domain of document management, a sub-domain that is part of (but not restricted to) product lines that have to deliver user or regulatory documentation accompanying product instances. The second article is not domain specific at all, but improves on ways how to generate variability models for legacy systems that missed to model and manage variability in the first place. A method and tool support for automatic configuration of feature models including preferences on functional as well as non-functional properties is presented in paper number three.

In their article "A Framework for Variable Content Document Generation with Multiple Actors", Abel Gomez Llana and his coauthors M. Carmen Penadésa, José H. Canósa, Marcos R. S. Borgesb and Manuel Llavadorc apply product line engineering techniques to motivate and design a method and an accompanying tool framework for managing product lines of documents. Distinguishing between support required for domain and for application engineering results in a well-structured methodology and tool framework. Like for every good product line approach, the authors start with analyzing the domain of document creation, the kinds of variability to be supported, the stakeholders involved. It always is the deep understanding of the domain at hand that drives product line engineering. And the choice of standards, open source tools and frameworks that helped the authors to build their framework shows a third important characteristic of product line engineering next to business driven and domain oriented: reuse of proven assets is a guiding principle, distinguishing the good product line engineer from the not-invented-here pioneer, leading to highest productivity in turning good ideas into usable products and tools.

Core to the framework for variable content document generation is the concept of feature modeling. A feature model represents the configurative variability of a product family, providing a way to understand, manage and use variability information for tasks like checking constraints on planned product instances and (semi)automatically deriving products from a set of product line core assets. The authors of "Efficient Synthesis of Feature Models", Andrezey Wasowsky and his colleagues Steven She, Uwe Ryssel, Nele Andersen and Krzysztof Czarnecki, are well known for their work in the area of feature modeling. Their article describes another step in making feature models as reasoning and product derivation tool available for legacy product lines. Legacy product lines tend accumulated variability over the years, making management of variability and product derivation and configuration cumbersome, error prone and thus expensive. Information mined from build systems, compiler flags and conditional compilation directives on the one hand, or from product configurations on the other is cast into propositional formulas that then is the input for feature model synthesis tools. However, computing feature models from such input is NP-hard, as Wasowski et.al. prove. Two algorithms for synthesis of feature models from propositional formulas in different normal forms are compared to existing approaches and show good results for large feature sets with many possible product configurations.

Feature models get enhanced with quality requirements in "Toward Automated Feature Model Configuration With Optimizing Non-Functional Requirements". Mohsen Asadi, Samaneh Soltani, Dragan Gasevic, Marek Hatala and Ebrahim Bagheri argue that, since non-functional requirements are as important as functional requirements for making the right choices at application engineering, support for automatic configuration considering those NFRs is extremely helpful. Quality requirements often influence each other in product lines. A product cannot fulfil all NFRs equally well, so it is important at configuration time to know the weight of NFRs, absolute and/or in relation to each other. To consider quantitative as well as qualitative NFRs and their interdependencies at automated configuration of feature models, the authors combine different techniques from in multi-criteria decision making and 
hierarchical planning. For proving the usefulness of the approach, the authors provide tooling for automated configuration and for reasoning over the respective results. Comparisons with similar

Christa Schwanninger Siemens AG, Germany

David Benavides* research tools show the competitiveness of the approach for reasonably sized feature models.
University of Seville, Spain

* Tel.: +34 954553866 .

E-mail address: splc2012specialsection@gmail.com

Available online 24 April 2014 